\title{
3DCRT Versus RapidArc in Terms of Iso-Dose Distribution, Dose Volume Histogram (DVH) and Organs at Risk for Esophageal Cancer (EC) Dosimetric Study
}

\author{
Saud. H. Allehyani ${ }^{1, ~ *}$, Huda. A. Sharyan ${ }^{1}$, Aida. R. Tolba ${ }^{1,2}, \operatorname{Rmadan}_{\text {. A. Hassan }}{ }^{1,2}$ \\ ${ }^{1}$ Physics Department, College of Applied Science, Umm ALQura University, Makkah, Saudi Arabia \\ ${ }^{2}$ Radiotherapy and Nuclear Medicine Department, National Cancer Institute, Cairo University, Cairo, Egypt
}

Email address:

saud8882001@yahoo.com (S. H. Allehyani)

*Corresponding author

\section{To cite this article:}

Saud. H. Allehyani, Huda. A. Sharyan, Aida. R. Tolba, Rmadan. A. Hassan. 3DCRT Versus RapidArc in Terms of Iso-Dose Distribution, Dose Volume Histogram (DVH) and Organs at Risk for Esophageal Cancer (EC) Dosimetric Study. American Journal of Clinical and Experimental Medicine. Vol. 5, No. 4, 2017, pp. 123-133. doi: 10.11648/j.ajcem.20170504.14

Received: May 10,2017; Accepted: May 19, 2017; Published: June 28, 2017

\begin{abstract}
Purpose: To compare (3D-CRT) to RapidArc planning using (LNAC of $6 \mathrm{MV}$ ) in terms of dosimetric outcomes of iso-dose distribution, dose volume histogram (DVH), PTV and at risk organs in 5 patients with Esophageal cancer (EC). Methods: Plans were created for 5 patients with EC who had received radical RapidArc treatment from 2012 to 2014 at KAMC (King Abdullah Medical City). Results: RapidArc plan showed a more homogeneous dose distribution in PTV, achieving an HI of $1.112 \pm 0.030$ compared with $1.096 \pm 0.029$ in the 3D-CRT plan. However, RapidArc and 3D-CRT achieved nearly equal in both modalities with average value of $(0.130 \pm 0.052)$ in RapidArc compared to $(0.134 \pm 0.054)$ in 3D-CRT, $(p=0.061)$ in terms of CI values and Target coverage index (TCI) was $(0.093 \pm 0.032)$ in RapidArcand and $(0.099 \pm 0.058)$ in 3D-CRT, $(p=$ 0.767). Additionally, regarding OARs, the mean and maximum dose in Spinal Cord was lower in RapidArc with a low percentage of the volume receiving low doses. Left and Right Lungs were within tolerance in RapidArc and 3D-CRT. RT. Kidney was lower in RapidArc, while LT Kidney was lower in 3D-CRT. For Heart, the maximum dose was within tolerance criteria in RapidArc but exceeded the criteria in 3D-CRT at 60.97 Gy. For the spinal cord, the maximum dose was notably low and did not exceed 1.91 Gy in RapidArc, while it exceeded the acceptable limit in 3D-CRT. Conclusions: (VMAT) is superior to 3D-CRT in term of PTV, conformity and homogeneity. This study suggests that VMAT class solution is the superior treatment option.
\end{abstract}

Keywords: Planning Tumor Volume, Organs at Risk, Conformity Index, Heterogeneity Index, Esophageal Cancer

\section{Introduction}

\subsection{Management of Patients with Cancer}

The optimal care of patients with malignant tumors is a multidisciplinary effort that combines classic modalities, surgery, radiation therapy, and chemotherapy. The role of the radiation oncologist is to assess all conditions relative to the patient and tumor, to systematically review the need for diagnostic and staging procedures, and, in consultation with other oncologists, determine the best therapeutic strategy. Radiation oncology includes the clinical and scientific discipline devoted to management of patients with cancer (and other diseases) with ionizing radiation (alone or combined with other modalities), investigation of the biologic and physical basis of radiation therapy, and training of professionals in the field. The aim of radiation therapy is to deliver a precisely measured dose of irradiation to a defined tumor volume with minimal damage to surrounding healthy tissue. This results in eradication of the tumor, increased quality of life, and prolongation of survival at a competitive cost, and allows for effective palliation or prevention of symptoms of cancer, including pain, restoring luminal patency, skeletal integrity, and organ function, with minimal morbidity $[1,2]$. 


\subsection{Process of Radiation Therapy}

The goal of therapy should be defined at the onset of therapeutic intervention: - Curative: There is a probability of long-term survival after adequate therapy. Some side effects of therapy, although undesirable, may be acceptable. • Palliative: There is no hope of survival for extended periods. Symptoms producing discomfort or an impending condition that may impair comfort or self-sufficiency require treatment. No major iatrogenic conditions should be observed. Relatively high doses of irradiation (sometimes $75 \%$ to $80 \%$ of the curative dose) are required to control the tumor for the survival period of the patient [3].

\subsection{Basis for Prescription of Irradiation}

The basis for Prescription of Irradiation includes Evaluation of the extent of the tumor (staging), including diagnostic studies, knowledge of pathologic characteristics of the disease, definition of the goal of therapy (cure or palliation) selection of appropriate treatment modalities (irradiation alone or combined with surgery, chemotherapy, or both). Determination of the optimal dose of irradiation and volume to be treated according to anatomic location, histologic type, stage, potential regional nodal involvement (and other tumor characteristics), and normal structures in the region. It also includes evaluation of the patient's general condition, plus periodic assessment of tolerance to treatment, tumor response, and status of normal tissues treated and Ultimate responsibility for treatment decisions, technical execution of therapy, and consequences of therapy always rests with the radiation oncologist $[1,4]$.

\subsection{Radiation Treatment Planning}

Different irradiation doses are required for various probabilities of tumor control, depending on the tumor type and the initial number of clonogenic cells present. Various radiation doses can be delivered to specific portions of the tumor periphery versus central portion) or to the tumor bed in cases in which the entire gross tumor has been surgically removed. The International Commission on Radiation Units and Measurements Reports Nos. 50 and 62 define the following treatment planning volumes $[5,6]$ : Gross tumor volume (GTV): all known gross disease, including abnormally enlarged regional lymph nodes. To determine GTV, appropriate computed tomography (CT) window and level settings that give the maximum dimension of what is considered potential gross disease must be used. Clinical target volume (CTV): Encompasses GTV plus regions considered to harbor potential microscopic disease. Planning target volume (PTV): provides margin around CTV to allow for internal target motion, other anatomic motion during treatment (e.g., respiration), and variations in treatment setup. PTV does not account for treatment machine beam characteristics [7]. Treatment portals must adequately cover all treatment volumes plus a margin to account for beam physical characteristics, such as penumbra. Simulation is used to accurately identify target volumes and sensitive structures and to document configuration of portals and the target volume to be irradiated. Treatment aids (e.g., shielding blocks, molds, masks, immobilization devices, compensators) are extremely important in treatment planning and delivery of optimal dose distribution. Repositioning and immobilization devices are critical because the only effective irradiation is that which strikes the clonogenic tumor cells [8]. Simpler treatment techniques that yield an acceptable dose distribution are preferred over more costly and complex ones, which may have a greater margin of error in day-to-day treatments. Accuracy is periodically assessed with portal (localization) films or on-line (electronic portal) imaging verification devices. Portal localization errors may be systematic or may occur at random [7].

\subsection{Three-Dimensional Treatment Planning}

CT simulation allows for a more accurate definition of target volume and anatomy of critical normal structures, three-dimensional (3-D) treatment planning to optimize dose distribution, and radiographic verification of the treated volume [9]. Advances in computer technology have augmented accurate and timely computation, display of 3-D radiation dose distributions, and dose-volume histograms that yield relevant information for the evaluation of tumor extent, definition of target volume, delineation of normal tissues, virtual simulation of therapy, generation of digitally reconstructed radiographs, design of treatment portals and aids, calculation of 3-D dose distributions and dose optimization, and critical evaluation of the treatment plan [10]. Dose-volume histograms are useful in assessing several treatment plan dose distributions and providing a complete summary of the entire 3-D dose matrix, and showing the amount of target volume or critical structure receiving more than the specified dose. They do not provide spatial dose information and cannot replace other methods of dose display [11]. 3-D treatment planning systems play an important role in treatment verification. Digitally reconstructed radiographs based on sequential CT slice data generate a simulation film that can be used in portal localization and for comparison with the treatment portal film for verifying treatment geometry [12]. Increased sophistication in treatment planning requires parallel precision in patient repositioning and immobilization, as well as in portal verification techniques. Several real-time, on-line verification systems allow monitoring of the position of the area to be treated during radiation exposure. Computer-aided integration of data generated by 3-D radiation treatment planning with parameters used on the treatment machine, including gantry and couch position, may decrease localization errors and enhance the precision and efficiency of irradiation [13].

\subsection{Intensity-Modulated Radiation Therapy}

Intensity-modulated radiation therapy (IMRT), a new approach to 3-D treatment planning and conformal therapy, optimizes delivery of irradiation to irregularly shaped volumes through complex forward or inverse treatment 
planning and dynamic delivery of irradiation that results in modulated fluency of multiple photon beam profiles. Inverse planning starts with an ideal dose distribution and identifies, through trial and error or multiple iterations (simulated annealing), the beam characteristics (fluence profiles). It then produces the best approximation of the ideal dose defined in a 3-D array of dose voxels organized in a stack of twodimensional arrays [12]. Other approaches to achieve IMRT include the step-and-shoot method, with a linear accelerator and multileaf collimation (MLC), which uses a variety of portals at various angles. The MLC determines photonmodulated fluency and portal shape by the dynamic computer-controlled IMRT being delivered when the configuration of the portals with the MLC changes at the same time that the gantry or accelerator changes positions around the patient.. In helical tomotherapy, a photon fan beam continually rotates around the patient as the couch transports the patient longitudinally through a ring gantry. The robotic arm IMRT system (Cyberknife) consists of a miniaturized MV photon linear accelerator mounted on a highly mobile arm and a set of ceiling-mounted x-ray cameras to provide near real time information on the patient's position and target exposure during treatment [14]. The majority of IMRT systems use $6 \mathrm{MV}$ x-rays, but energies of 8 to $10 \mathrm{MV}$ may be more desirable in some anatomic sites to decrease skin and superficial subcutaneous tissue dose [15].

\subsection{Quality Assurance}

A comprehensive quality assurance (QA) program is critical to ensure the best treatment for each patient and to establish and document all operating policies and procedures. QA procedures in radiation therapy vary, depending on whether standard treatment or a clinical trial is carried out, and if such treatments and trials occur at single or multiple institutions. In multi-institutional studies, it is important to provide all participants with clear instructions and standardized parameters in dosimetry procedures, treatment techniques, and treatment [16].

\subsection{Principles of IMRT}

The concept of IMRT was not applied until the 1990s. The software and hardware were not available before that time [17]. IMRT is a more advanced mode of conformal radiotherapy and an extension of 3-dimensional conformal radiation therapy (3D-CRT) that includes the use a larger number of $\mathrm{x}$-ray beam compared to 3D-CRT. Therefore, large volumes of healthy tissue are exposed to low levels of radiation $[18,19]$. IMRT allows for appropriate conforming of the high and low doses to the target and healthy tissue, by creating non-uniform radiation beam intensities across the irradiation field. This creation can be performed in two ways: step and shoot (static technique) or sliding window (dynamic technique) $[14,19,20]$. Intensity modulated arc therapy (IMAT) is the next step in IMRT radiation delivery, whereby the gantry rotates around the patient and the radiation dose is delivered continuously in an arc [14]. It is possible to summarize the advantages of IMRT in good sparing to critical structures and fairly quick planning. However, the disadvantages include complex QA and longer treatment time.

\subsection{Volumetric Modulated Arc Therapy (VMAT)}

Volumetric modulated arc therapy (VMAT) is a novel from of IMRT that allows the radiation to be delivered to the patient in a single $360^{\circ}$ of gantry rotation that is accurately and efficiently with varying velocities and positions of the MLC, dose rate and gantry speed. This leads VMAT being an intensity-modulated dose distribution [21]. RapidArc (Varain medical system) is a form of VMAT [22]. RapidArc (RA) is intended to protect healthy tissue more than other techniques and to improve target coverage distribution and treatment time, and attain accurate dosimetric delivery to have the ideal dose distribution. VMAT has many different advantages over conventional modality 3D-CRT [20]. The fundamental feature is treatment time. VMAT treatment time was 1.3 minutes, IMRT treatment time was 8 minutes and 3D-CRT was 3 minutes [23,]. Other studies have demonstrated a similar decline in treatment time between VMAT and 3DCRT. Depending on decreased treatment time in the machine, patient comfort, compliance and throughput increased. The main disadvantage of VMAT is the increased optimization time compared to 3D-CRT. Shannon M. MacDonald et al. [24] compared 3D-CRT with IMRT for 20 patients treated for high - grade glioma. The prescribed dose was 59.4 Gy. The authors showed that IMRT was superior in target coverage compared with 3D-CRT plans, and effectively reduced radiation dose to the brain, brain stem and optic chiasm. David Palam et al. [21] compared three techniques: VMAT, IMRT and 3D-CRT for 10 patients with prostate cancer. The comparison showed lower doses to normal critical structure and achieved highly conformal treatment plans in VMAT and IMRT over 3D-CRT plans. Luca Cozzi et al. [25] used a treatment planning system to compare Volumetric Arc Modulation with RapidArc and IMRT for cervix uteri of 8 patients. Both RA and IMRT showed equivalent target coverage. RA improved CI, HI and OARs sparing. Wilko F. A. R Verbakel et al. [26] compared VMAT with conventional IMRT in 12 patients for head and neck cancer. The comparison showed that double arc plans provided at least similar sparing of OAR and better PTV dose homogeneity than single arc or IMRT. Bao-min Zheng et al. [27] compared VMAT with RA and fixed filed dynamic IMRT for 20 patients with nasopharyngeal carcinoma. The prescribed dose was $70 \mathrm{~Gy}$. For both RA and IMRT, the target volume coverage was similar. Peszynska-piorun et al. [28] compared IMRT with 3D-CRT for 25 patients with head and neck cancer. IMRT delivered comparable or greater doses to OARs, while 3D-CRT was found to better spare the organs. Andrea Holt et al. [29] compared VMAT with IMRT in 5 oropharyngeal cancer patients from five institutes. VMAT showed significantly better sparing of OARs and improvement in CI. Kham Nguyen et al. [30] compare VMAT versus IMRT in 10 nasal cavity patients and found 
that VMAT achieved similar or better target coverage in comparison to IMRT. VMAT plans better spared critical structure and nearly normal tissue from higher dose volumes. IMRT plans were favored in minimizing the volume of low dose received to normal tissue and critical structures. Nima Nabavizadeh et al. [31] compared VMAT with 3D-CRT and IMRT in 20 patients with pancreatic cancer. VMAT and IMRT were shown to be superior over 3D-CRT in planning target volume (PTV) coverage and sparing OARs.

\section{Material and Methods}

\subsection{Equipment Used}

\subsubsection{Linear Accelerator}

The linear accelerator utilized for treatment planning was the Trilogy equipped with the Millennium Multi leaf Collimator by Varian Medical Systems. It is able to deliver beams of electrons and photons. Only the photon beam is used in this study for all cases with energies of $6 \mathrm{MV}, 15 \mathrm{MV}$ and $18 \mathrm{MV}$. There are 120 leaves total with 40 leaf pairs in the center and 10 pairs on each side. The center leaf width is $5 \mathrm{~mm}$ projected at isocenter, while the outer leaves are larger at $10 \mathrm{~mm}$. The maximum leaf speed is $2.5 \mathrm{~cm} / \mathrm{s}$. The treatment planning system was the external beam planning system of Eclipse (Version 10.0.28.2, Varian Medical System) and the volume calculation used was the Anisotropic Analytical Algorithm (AAA, version 10.0.28.2). The Progressive Resolution Optimizer (PRO) utilized in the RapidArc optimization was Version 10.0.28.2. Varian's Leaf Motion Calculator (version 10.0.28.2) was enabled for the IMRT leaf sequence generation (see figure 1.).

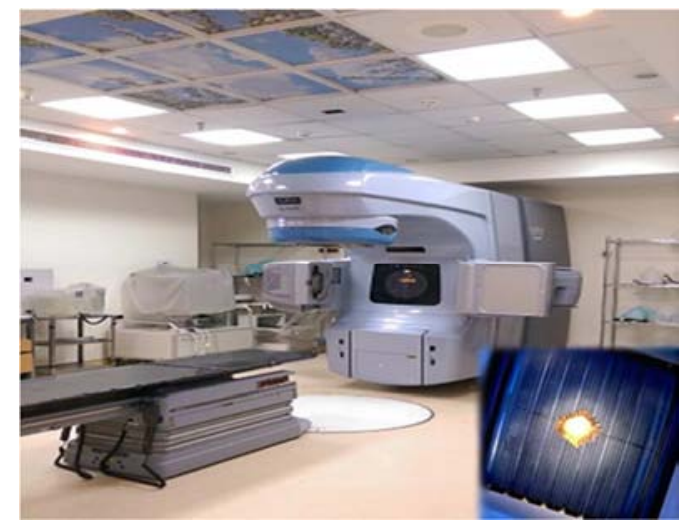

Figure 1. LINAC machine and $M L C$.

\subsubsection{CT Simulation}

It is necessary for each cancer center to have CT simulator in the radiation therapy department [32]. CT scan should include the part of the body to be treated with radiation. A slice thickness between $3 \mathrm{~mm}$ and $5 \mathrm{~mm}$ is recommended for CT scanning except for head and neck, which may be between $2 \mathrm{~mm}$ and $3 \mathrm{~mm}$ [34]. The CT scanner couch should be flat and comfortable for the patient and compatible with the therapy machine couch. The positioning of laser lights in the $\mathrm{CT}$ room must be similar to those in the treatment room to ensure exact positioning of the patient (see figure 2). In this study, the patients underwent pervious computed tomography simulation (CT-sim) (GE Light Speed 16 Slice $\mathrm{CT}$ ) for treatment planning. For all patients, plans were designed on CT scans acquired $5 \mathrm{~mm}$ slice thickness, except for head and neck cases that acquired $2 \mathrm{~mm}$ slice thickness, and included the region of interest. The patients were positioned supine and straight and level.

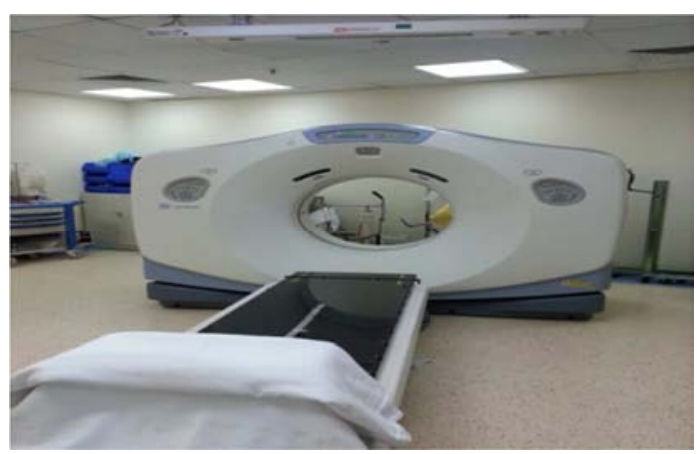

Figure 2. CT scanner.

\subsection{Planning Technique}

After simulation, the CT images were transferred to the External Beam planning system of Eclipse using $6 \mathrm{MV}$ and 15 MV photon beam data. The Progressive Resolution Optimizer (PRO) was used for the RapidArc plans. The Anisotropic Analytical Algorithm (AAA) was used for photon dose calculation for all cases. For RapidArc, arcs were used clockwise $\left(181^{\circ}-179^{\circ}\right)$ and anticlockwise $\left(179^{\circ}-181^{\circ}\right)$, the collimator was rotated $30^{\circ}$ to $330^{\circ}$ with the dose rate varied between $0 \mathrm{MU} / \mathrm{min}$ and $600 \mathrm{MU} / \mathrm{min}$ (upper limit) to reduce the effect due to inter-leaf leakage. The double arc technique was expected to achieve better target dose coverage than the single arc because the independent optimization of two arcs allows each arc to create a completely unrelated sequence of MLC shapes, dose rates and gantry speed combinations. For the 3DCRT plans, all of the gantry angles and numbers of radiation fields (range, 3-4) were manually selected on the basis of the formalism relationship between the PTV and OARs to cover at least $95 \%$ of the PTV and spare the OARs. The dose rate of 400 $\mathrm{MU} / \mathrm{min}$ was used for 3D-CRT. Wedges were used to provide a more homogenous distribution. The optimization constraints for OARs using RapidArc are illustrated in table 1.

Table 1. The dose constraints of organ at risk.

\begin{tabular}{lll}
\hline $\begin{array}{l}\text { Critical organ at } \\
\text { risk }\end{array}$ & First Criteria: Ideal & $\begin{array}{l}\text { Second Criteria: } \\
\text { Acceptable }\end{array}$ \\
\hline Spinal cord & Max dose $\leq 45 \mathrm{~Gy}$ & Max dose $\leq 45 \mathrm{~Gy}$ \\
Liver & Mean dose $<30-32$ Gy & Max dose $\leq 45$ Gy \\
Heart & Mean dose $<26$ Gy & Max dose $\leq 45$ Gy \\
Kidney & V30 $<46 \%$ & Max dose $\leq 45$ Gy \\
Lung & V25 $<10 \%$ & Max dose $\leq 45$ Gy \\
\hline
\end{tabular}

Spinal cord is set-up to have a maximum dose limit, both of liver and kidneys are set up to have dose constrain corresponding to the mean dose. V20Gy, V10Gy and V5Gy are to evaluate the lungs and mean dose, V30Gy, V15Gy and 
V5Gy were also compared. The upper and lower limits on the PTV are set to $107 \%$ and $95 \%$ of the prescribed dose respectively.

\subsection{Compile Patient Database}

\subsubsection{Patient Selection}

Institutional Review Board (IRB) approval was obtained before the initiation of this retrospective study. The plans of five different malignant tumor patients who had received radical RapidArc treatment from 2012 to 2014 at KAMC were randomly selected and re-planned for 3-dimensional conformal radiation therapy. The sample included both male and female patients and the median age was 54 years old (range, 47 - 57 Years).

\subsubsection{Patient Anonymization}

Patient names, age, sex, treatment site, treatment modality and codes were collected and recorded in an Excel sheet. Each patient was assigned a research code of $0 \mathrm{xx}$, where $\mathrm{xx}$ is a number from 01 to 05 . The patient's last name and medical record number were replaced by this research code, and all other personal information was removed. Furthermore, the personal information in the image set header files was removed. Table 2. lists the cases used for this study, indicating their age, sex, treatment site and modality. A malignant neoplasm is composed of cells that look less similar to the normal cell of origin or an abnormal mass of tissue arising from an abnormal proliferation of the cells. Malignant neoplasms derived from epithelial cells are called carcinomas, which is a cancer that begins in the skin or in tissue that cover body organs. Those derived from mesenchymal (connective tissue) cells are called sarcomas. Malignant brain neoplasms and neoplasms of the immune system are special categories with complex nomenclature [34].

Table 2. Patient database (Malignant neoplasm).

\begin{tabular}{|c|c|c|c|c|}
\hline Code & Sex & Age & Treatment site & Modality \\
\hline 001 & $\mathrm{~F}$ & 63 & $\begin{array}{l}\text { Malignant neoplasm of } \\
\text { lower third of esophagus }\end{array}$ & RA - 3DCRT \\
\hline 002 & $\mathrm{~F}$ & 46 & $\begin{array}{l}\text { Malignant neoplasm of } \\
\text { lower third of esophagus }\end{array}$ & RA - 3DCRT \\
\hline 003 & M & 57 & $\begin{array}{l}\text { Malignant neoplasm of } \\
\text { abdominal esophagus }\end{array}$ & RA - 3DCRT \\
\hline 004 & M & 62 & $\begin{array}{l}\text { Malignant neoplasm of } \\
\text { lower third of esophagus }\end{array}$ & RA - 3DCRT \\
\hline 005 & $\mathrm{~F}$ & 63 & $\begin{array}{l}\text { Malignant neoplasm of } \\
\text { lower third of esophagus }\end{array}$ & RA - 3DCRT \\
\hline
\end{tabular}

\subsection{Treatment Plan Evaluation Metrics}

\subsubsection{Dosimetric Plan Evaluation Metrics}

The dosimetric evaluation metrics used to compare the two plans, in terms of mean, maximum and minimum doses to PTV, were dose to $95 \%$ of PTV, Homogeneity Index (HI), Conformity Index (CI), Target Coverage Index (TCI) and Mean and maximum doses to critical organs and normal tissue. The dose to $95 \%$ of the PTV (D95\%) was used to quantify PTV coverage. The homogeneity index (HI) was used to evaluate uniformity (homogeneity) of dose within the PTV and is calculated as

$$
H=\frac{D_{5}}{D_{95}}
$$

Where $D_{5}$ and $D_{95}$ represent the dose delivered to $5 \%$ and 95\% of the PTV, respectively. The smaller and closer the value of $\mathrm{HI}$ is to 1 , the better the homogeneity of the PTV [35]. The conformity index (CI) was also calculated and can be defined as the degree of conformity of the plans, which is a ratio of the PTV receiving 95\% of the prescribed dose divided by the volume of the PTV. A CI value approaching 1 indicates a higher degree of conformity.

$$
C I=\frac{P T V_{95} \% P D}{V_{P T V}}
$$

The target coverage index (TCI) accounts for the exact coverage of PTV in the treatment plan at the prescribed dose as shown below:

$$
T C I=\frac{P T V_{P D}}{P T V}
$$

Where $\mathrm{PTV}_{\mathrm{PD}}$ is the PTV coverage at the prescribed dose (PD) and PTV is the volume of PTV. Target conformity index reports target dose coverage as a value between 0 and 1 . A value of 1 indicates an ideal plan with target coverage by prescribed dose. However, a TCI value of 0 indicates the whole target volume is not covered by the prescribed dose $[36,37,38]$.

\subsubsection{Esophageal Cancer (EC)}

Esophageal cancer (EC) remains one of the most aggressive and lethal digestive diseases world-wide. It is associated with poor outcomes and presents a challenge to surgeons, doctors, and ra-diation oncologists. There are approximately 16,000 newly diagnosed patients with EC each year, and an estimated 14,000 patient deaths were reported in the United States in 2008 [39]. Squa-mous cell carcinoma is commonly seen in Asian countries, whereas adenocarcinoma is common in Europe and America. Most EC patients are at an advanced stage or are unresectable at the time of initial diagnosis [40]. Concurrent chemoradiother-apy (CCRT) is the major treatment method for local advanced or unresectable esophageal cancer, but the 5 -year overall survival rate is only $15 \%$ to $25 \%$ [41]. Local failure is the most common failure pattern associated with CCRT, and local persis-tence of the disease occurs in $60 \%$ to $70 \%$ of pa-tients [42]. The results of the Radiation Therapy Oncology Group 94-05 trial demonstrated few survival benefits for the group receiving a higher dose of radiation therapy [43]. However, the investigators used a traditional 2-dimensional (2D) technique with anteroposterior/posteroanterior (AP/PA) field arrangement to deliver radiotherapy (RT), which limited the dose provided to the tumor because of concerns about the safety of the surrounding healthy tissue. Studies have shown that the use of modern RT techniques is needed to clarify the possible benefits of dose escalation. Intensity-modulated 
radiotherapy (IMRT) constitutes an important advance in techniques for improving tumor coverage and reducing the doses delivered to the surrounding normal tissues. IMRT is superior to $3 \mathrm{D}$ conformal radiotherapy (3D-CRT) or 2D-RT based on dosimetric analysis [44, 45]. Volumetric modulated arc therapy (VMAT), a novel form of IMRT that was first proposed by $\mathrm{Yu}$ in 1995[46]. is a widely used radiation technique and is regarded as a new generation linear-accelerator IMRT. VMAT can promote the delivery of a substantial radiation dose to the tumor while avoiding the delivery of an excess dose to the healthy tissues in the tumor vicinity. Moreover, VMAT can produce plans that are dosimetrically equivalent to IMRT for centrally located cancers such as cancers of the anal canal, prostate cancer, cervical cancer, and head and neck cancers [4749]. A treatment planning study was performed to evaluate the performance of volumetric arc modulation with RapidArc (RA) against 3D conformal radiation therapy (3D-CRT) and conventional intensity-modulated radiation therapy (IMRT) techniques for esophageal cancer [50]. In this study, we compared VMAT and 3D conformal radiotherapy (3D-CRT) for patients with EC in terms of PTV, HI, CI and TCI in all locations with respect to the dose distributions, planning target volumes (PTVs), and organs at risk (OARs). Five patients of esophagus cancer whose received 50.4 Gy in 25 fractions were discussed in this study. The median age was 49 years. CT Scans with $0.5 \mathrm{~cm}$ slice thickness used for planning. After CT scan, TPS import the images to initiate the planning. RapidArc plan was used two arcs rotating clockwise and anticlockwise, gantry angles $\left(181^{\circ}-179^{\circ}\right)$ and collimator angles $\left(30^{\circ}-330^{\circ}\right)$, using $6 \mathrm{MV}$ photon and dose rate 600 $\mathrm{MU} / \mathrm{min}$. After the CT scan, the images were transferred to the treatment planning system (TPS) to initiate the planning. RapidArc plan was used two full arcs, the first arc rotating in a clockwise direction from $181^{\circ}$ to $179^{\circ}$ with a collimator angle of $30^{\circ}$. The second arc rotated in the opposite direction from $179^{\circ}$ to $181^{\circ}$ with a collimator angle of $330^{\circ}$ (see figure 2), using $6 \mathrm{MV}$ and a maximum dose rate of $600 \mathrm{MU} / \mathrm{min}$. The arcs moved in opposite directions to Representation of patients' prescription doses, PTV volume, and number of fields (no/f) for both 3D-CRT and RA, are shown in table 3.

Table 3. Patients' prescription doses, PTV volume, and number of fields for both $3 D-C R T$ and $R A$.

\begin{tabular}{lllllll}
\hline Pc & PD(Gy) & No of $\mathbf{F}$ & D/F & PTV(cm3) & \multicolumn{2}{l}{ No of F/A } \\
\hline & & & & & RA & 3D-CRT \\
\hline 001 & 50.4 & 25 & 2 & 638 & 2 arcs & 4 fields \\
002 & 50.4 & 25 & 2 & 564.6 & 2 arcs & 4 fields \\
003 & 45 & 25 & 1.8 & 2098.4 & 2 arcs & 4 fields \\
004 & 50.4 & 25 & 2 & 818.2 & 2 arcs & 4 fields \\
005 & 50.4 & 25 & 2 & 547.3 & 2 arcs & 4 fields \\
\hline
\end{tabular}

\section{Results}

Differences were recorded between those patients who planned with 3D-CRT and those who planned with
RapidArc. Thus one patient was selected to represent all other patients in this site for isodose distribution comparison, dose volume histogram (DVH) comparison, dosimetric results for the PTV and dosimetric results for the critical organs. DVHs figures include the PTV and critical organs for each modality and show the percentage of the total volume (y-axis) of each ROI receiving a specified dose (x-axis) in units of Gy. Table 4 shows the mean, max and minimum dose that covered $95 \%$ of the target and p-value of the target (PTV) for both modalities. The prescribed dose was 50.4 Gy and table.5. show the evaluation metrics for the PTV in terms of CI, HI and TCI.

Table 4. Evaluation metrics for PTV in terms of. $D_{M E A N}, D_{\max }$ and $D_{\min }$ covered $95 \%$ of the target, Pc (patient code).

\begin{tabular}{|c|c|c|c|c|c|c|c|c|}
\hline \multirow{3}{*}{$\begin{array}{l}\text { Pc } \\
001\end{array}$} & \multirow{2}{*}{\multicolumn{2}{|c|}{$\begin{array}{l}D_{\text {mean }}(G y) \\
\text { RA - 3DCRT }\end{array}$}} & \multirow{2}{*}{\multicolumn{2}{|c|}{$\begin{array}{l}D_{\max }(G y) \\
R A-3 D C R T\end{array}$}} & \multirow{2}{*}{\multicolumn{2}{|c|}{$\begin{array}{l}D_{\min }(G y) \\
R A \\
\text { 3DCRT }\end{array}$}} & \multirow{2}{*}{\multicolumn{2}{|c|}{$\begin{array}{l}\text { D }_{95 \%}(\mathrm{~Gy}) \\
\text { RA 3DCRT }\end{array}$}} \\
\hline & & & & & & & & \\
\hline & 51.1 & 51.1 & 56.9 & 55.7 & 34 & 37.8 & 46 & 48.0 \\
\hline 002 & 50.6 & 50.6 & 53.3 & 55.6 & 43 & 43.9 & 48 & 50.4 \\
\hline 003 & 46.5 & 46.5 & 50.6 & 49.8 & 31 & 36.4 & 43 & 43.9 \\
\hline 004 & 50.9 & 50.9 & 55.9 & 52.1 & 37 & 44.6 & 48 & 48.3 \\
\hline 005 & 50.9 & 50.9 & 57.2 & 54.6 & 43 & 44.0 & 47 & 48.9 \\
\hline Mean & 50.0 & 50.26 & 54.8 & 53.56 & 38 & 41.34 & 47 & 47.90 \\
\hline $\begin{array}{l}\text { P- } \\
\text { value }\end{array}$ & \multicolumn{2}{|c|}{$\mathrm{P}<0.636$} & \multicolumn{2}{|c|}{$\mathrm{P}<0.301$} & \multicolumn{2}{|c|}{$\mathrm{P}<0.050$} & \multicolumn{2}{|c|}{$\mathrm{P}<0.064$} \\
\hline
\end{tabular}

Table 5. Evaluate metrics for the PTV.

\begin{tabular}{lllllll}
\hline Pc & $\mathbf{C I}=\frac{P T V_{\mathbf{9 5}} \boldsymbol{P D}}{\boldsymbol{V}_{\boldsymbol{P T V}}}$ & \multicolumn{2}{l}{$\mathbf{H I}=\frac{\boldsymbol{D}_{\mathbf{5}}}{\boldsymbol{D}_{\mathbf{9 5}}}$} & & \multicolumn{2}{l}{ TCI $=\frac{P T V_{\boldsymbol{P D}}}{\boldsymbol{P T V}}$} \\
\hline & RA & 3DCRT & RA & 3DCRT & RA & 3DCRT \\
\hline 001 & 0.144 & 0.1501 & 1.141 & 1.1404 & 0.1218 & 0.1092 \\
002 & 0.172 & 0.1767 & 1.0736 & 1.0753 & 0.1156 & 0.1690 \\
003 & 0.046 & 0.0467 & 1.114 & 1.1056 & 0.0411 & 0.0368 \\
004 & 0.118 & 0.1192 & 1.089 & 1.0653 & 0.0893 & 0.0440 \\
005 & 0.170 & 0.1800 & 1.1421 & 1.0975 & 0.1017 & 0.1386 \\
Mean & 0.130 & $0.134 \pm$ & $1.11 \pm$ & $1.096 \pm$ & $0.09 \pm$ & $0.099 \pm$ \\
P-value & \pm 0.05 & 0.054 & 0.030 & 0.029 & 0.032 & 0.058 \\
\hline
\end{tabular}

\subsection{Planning Target Volume (PTV)}

No statistically significant difference between RapidArc and 3D-CRT in the mean dose to the PTV $(p<0.636)$ has been observed. The mean value of the PTV was $50.0 \pm 1.96$ in RapidArc and $50.26 \pm 2.60$ in 3D-CRT. The maximum dose to the PTV in RapidArc $(54.78 \pm 2.79)$ and in 3D-CRT $(53.56 \pm$ $2.55)$ had a lower maximum dose to the PTV $(p=0.301)$. This results indicates that RapidArc was better than 3D-CRT. The average minimum dose in RapidArc was $(37.86 \pm 5.36)$ compared to $(41.34 \pm 3.91)$ in 3D-CRT, $(p=0.050)$. The dose to $95 \%$ of the PTV was $(46.94 \pm 1.99)$ in RapidArc to $(47.90 \pm$ $2.41)$ in 3D-CRT, $(p=0.064)$. Conformity index $(\mathrm{CI})$ was approximately equal in both modalities with an average value of $0.626 \pm 0.695$ in RapidArc compared to $(0.130 \pm 0.052)$ in $3 \mathrm{D}$ CRT compared to $(0.134 \pm 0.054)$ in 3D-CRT, $(\mathrm{p}=0.061)$. The average homogeneity index (HI) in VMAT was $1.112 \pm 0.030$ to $1.096 \pm 0.029$ in 3D-CRT, $(p=0.146)$. Therefore, RapidArc achieved an improvement in HI. Target coverage index (TCI) in RapidArc was $0.093 \pm 0.032$ and $0.099 \pm 0.058$ in $3 \mathrm{D}-\mathrm{CRT}(\mathrm{p}=$ 0.767). 


\subsection{Patient-002}

Patient-002 was a 46-year-old woman diagnosed with a Malignant neoplasm of lower third of esophagus. After receiving curative dose by RapidArc, 3D-CRT plan was selected for the comparison.

\subsubsection{Isodose Distribution Comparison}

Isodose distributions for the RapidArc are displayed in figure $3 \mathrm{~A}$ and $3 \mathrm{D}-\mathrm{CRT}$ in figure $3 \mathrm{~B}$. The 3D-CRT plan contained the PTV receiving greater than $110 \%$ of the prescription dose, 55.4 Gy. This was not the case in the RapidArc plan, as the dose distribution within the PTV was more homogeneous. There were hot spots (doses greater than 54 Gy) in the lateral portion of the PTV in the 3D-CRT plan and in the upper portion of the PTV in the RapidArc plan. The distributions showed comparable PTV dose coverage between the two modalities. PTV conformity in the 3D-CRT plan appeared worse than in RapidArc. The 25 Gy lines extended farther to cover a large part of treatment area in the 3D-CRT plan than in RapidArc. However, a small region of the PTV in the 3D-CRT, RapidArc plans receiving 55 Gy, 56 Gy or greater, respectively. Demonstrating in what appeared to be greater PTV dose conformity in the RapidArc plan.

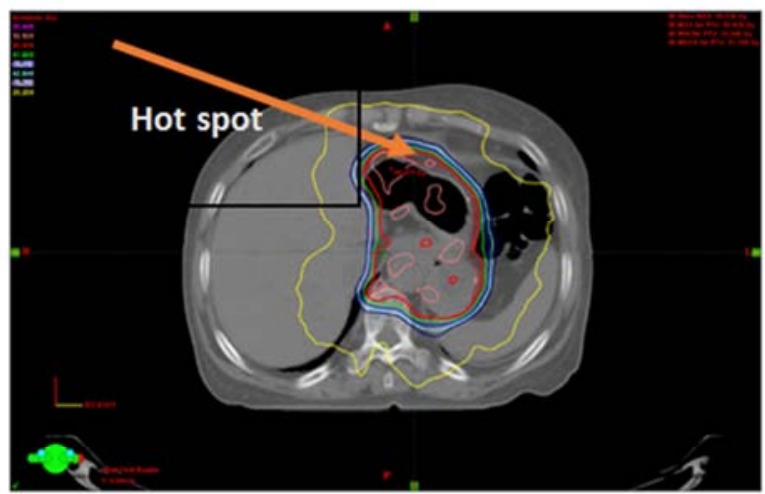

A

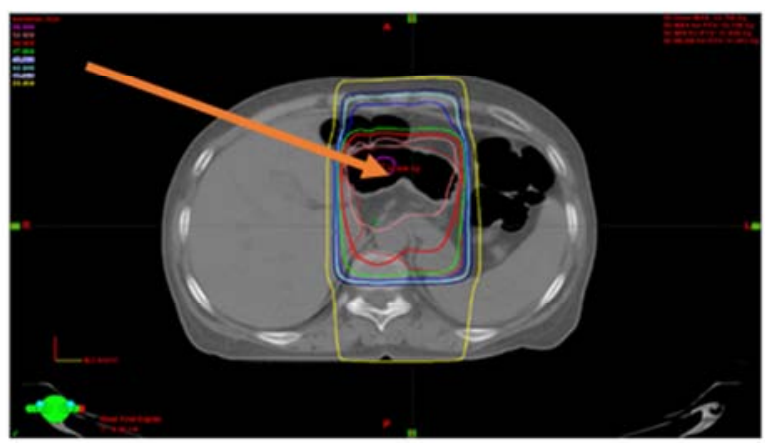

B

Figure 3. Isodose distributions for patient-002 showing (A) RapidArc and (B) $3 D-C R T$.

PTV conformity in the RapidArc plan was better than in the 3D-CRT. The 25 Gy lines extended farther to include the whole regionin the $3 \mathrm{D}-\mathrm{CRT}$ plan. There was a small region of the PTV in the 3D-CRT plan receiving 54 Gy or greater, resulting in what appeared to be greater PTV dose conformity in the RapidArc plan.

\subsubsection{DVH Comparison}

DVH provides useful quantitative dose assessment by direct visual inspection of the dose curve [49]. Figure 4 contains a DVH for the RapidArc and 3D-CRT plans for patient-002. The y-axes of a DVH, specifically for the PTV, represent the region where the curve bends away from $100 \%$ and "falls off" with the curve maintaining a constant slope. The RapidArc plan contained a broader region in the PTV, which indicates higher dose coverage compared with 3DCRT. The PTV had a sharper falloff in the RapidArc plan representing the superior PTV dose homogeneity observed in the isodose distributions. DVHs showed a higher dose to optic chiasm, optic nerve, left and right lens and left eye in the RapidArc plan comparable to that of 3D-CRT, and a low dose to the brain stem, spinal cord, right eye and right optic nerve in RapidArc.

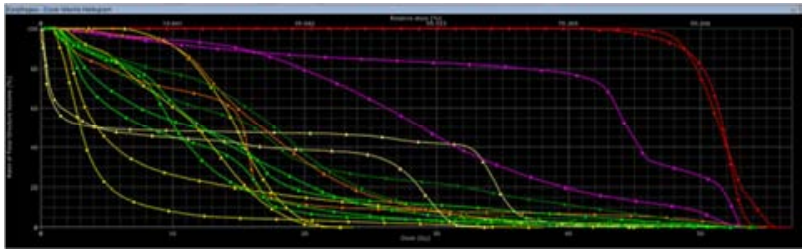

Figure 4. Comparison of DVHs between RA (triangles) and $3 D-C R T$ (squares). The PTV in red, heart in purple, kidneys in dark yellow, liver in orange, lunge in green and spinal cord light yellow.

Table 6. Evaluation metrics for the PTV-patient-002.

\begin{tabular}{llll}
\hline Parameter & Objective & RA & 3D-CRT \\
\hline Mean (Gy) & 50.4 & 50.6 & 52.6 \\
$\mathrm{D}_{\max }(\mathrm{Gy})$ & 53.928 & 53.3 & 55.6 \\
$\mathrm{D}_{\min }(\mathrm{Gy})$ & 47.88 & 43.5 & 43.9 \\
$\mathrm{D}_{95 \%}$ & 47.88 & 48.4 & 50.4 \\
Conformity Index (CI) & 1 & 0.1724 & 0.1767 \\
Homogeneity Index (HI) & 1 & 1.0736 & 1.0753 \\
Target Conformity Index (TCI) & 1 & 0.1156 & 0.1690 \\
\hline
\end{tabular}

Although the PTV has better coverage in 3D-CRT but the OARs achieved unacceptable overdose to the heart with dose improvement to other healthy structure in RapidArc. Results for the PTV (Patient 002) are shown in table 6. The RapidArc plan showed better dosimetric results in the PTV metric for patient-002. The RapidArc plan achieved a lower mean and maximum dose to the PTV. PTV dose coverage, as measured by the minimum dose and the dose to $95 \%$ of the volume, was higher in the RapidArc plan. The RapidArc plan also showed a more homogeneous dose distribution in the PTV, achieving an HI of 1.073 compared with 1.075 in the 3DCRT plan. However, However, the RapidArc plan was slightly higher CI than 3D-CRT and TCI had value higher in RapidArc compared to 3D-CRT.

\subsubsection{Organs at Risks (OARs)}

From table 7, DVHs explained the improvement in the dose that reached the spinal cord using RapidArc of 30.9 Gy, 
where it was almost up to the acceptable limit with 3D-CRT of $44.8 \mathrm{~Gy}$. The mean dose to the liver was found to be lower in 3D-CRT of 15.4 Gy compared to RapidArc of 17 Gy. The dose to right kidney was 2.1 Gy was lower in 3D-CRT comparable to 7.5 Gy in RapidArc and left kidney in 3DCRT has 15.5 Gy to 11.3 Gy in Rapidarc. V30Gy in the heart was lower in the RapidArc plan. The maximum dose to the heart was lower in the RapidArc plan, which received a dose of 52.8 Gy compared with a maximum of 54.7 Gy in 3DCRT. The entire heart received greater than 5 Gy in the RapidArc plan 97\%, while only $96.4 \%$ received this dose in 3D-CRT. Similarly, $90.0 \%$ of the heart received greater than 15 Gy in the RapidArc plan, while $88.8 \%$ received this dose in 3D-CRT. The mean dose to the heart was 24.8 Gy in the RapidArc plan compared with 28.2 Gy in 3D-CRT. For right lung, the mean dose was lower in 3D-CRT, but maximum dose was lower in RapidArc. V20Gy in the right lung was lower in the 3D-CRT plan; however, a larger percentage of the volume of the right lung received low doses in the RapidArc plan. $84.2 \%$ of the right lung received at least $5 \mathrm{~Gy}$ in the RapidArc plan, while only $61.7 \%$ received this dose in the 3D-CRT plan. Similarly, $10.2 \%$ of the right lung received greater than 10 Gy in the RapidArc plan, while only $7.2 \%$ received this dose in the 3D-CRT plan. For left lung, the mean dose was lower in 3D-CRT, but maximum dose was lower in RapidArc. V20Gy in the left lung was lower in the 3D-CRTplan; however, a larger percentage of the volume of the right lung received low doses in the RapidArc plan. $86.6 \%$ of the left lung received at least 5 Gy in the RapidArc plan, while $82.4 \%$ received this dose in the $3 \mathrm{D}$-CRT plan. Similarly, $77.8 \%$ of the left lung received greater than 10 Gy in the RapidArc plan, while $64.4 \%$ received this dose in the 3D-CRT plan. Finally, the mean dose were equal in both techniques, maximum dose to spinal cord was better with 2 Gy difference in RapidArc over 3D-CRT in liver. Right kidney was lower in 3D-CRT while lower in left kidney in RapidArc, The dose to heart was better un RapidArc and the mean dose in right lung were equal, V5Gy, V10Gy and V20Gy were lower in 3D-CRT. In left lung, the mean dose, V5Gy, V10Gy and V20Gy were lower in 3D-CRT. Generally, RapidArc had better sparing of OARs.

Table 7. Evaluation metrics for the OARs - patient-002.

\begin{tabular}{|c|c|c|c|c|}
\hline Organ & Parameter & Objective & $\mathbf{R A}$ & 3D-CRT \\
\hline Spinal Cord & Dmax (Gy) & $\leq 45$ & 30.9 & 44.8 \\
\hline Liver & Mean (Gy) & $<30-32$ & 15.4 & 17 \\
\hline RT. Kidney & Mean (Gy) & $<15-18$ & 7.5 & 2.1 \\
\hline LT. Kidney & Mean (Gy) & $<15-18$ & 11.3 & 15.5 \\
\hline \multirow{4}{*}{ Heart } & Mean (Gy) & $<26$ & 24.8 & 28.2 \\
\hline & V5Gy & No more than $5 \%$ of the heart for left-sided cancer & 97.0 & 96.4 \\
\hline & V15Gy & No more than $15 \%$ of the heart for left-sided cancer & 90.0 & 88.8 \\
\hline & V30Gy & No more than $30 \%$ of the heart for left-sided cancer & 45.4 & 82.7 \\
\hline \multirow{4}{*}{ RT. Lung } & Mean (Gy) & Minimize & 15.3 & 15.1 \\
\hline & V5Gy & No more than $50 \%$ of the lung for right-sided cancer & 84.2 & 61.7 \\
\hline & V10Gy & No more than $35 \%$ of the lung for right-sided cancer & 51.4 & 45.1 \\
\hline & V20Gy & No more than $15 \%$ of the lung for right-sided cancer & 10.2 & 7.2 \\
\hline \multirow{4}{*}{ LT. lung } & Mean (Gy) & Minimize & 13.1 & 10.3 \\
\hline & V5Gy & No more than $50 \%$ of the lung for left-sided cancer & 86.5 & 82.4 \\
\hline & V10Gy & No more than $35 \%$ of the lung for left-sided cancer & 77.8 & 64.4 \\
\hline & V20Gy & No more than $15 \%$ of the lung for left-sided cancer & 36.7 & 31.9 \\
\hline
\end{tabular}

\section{Discussion}

Comparison was performed by dose-based analysis on PTV range and critical organs. CI, HI and TCI were calculated. Nearly all of the dosimetric planning goals were met in the VMAT plans for each of the five patients in this study and are explained individually. As a baseline for the study, treatment plans were created using two full gantry rotation arcs. Several studies have found that the use of two arcs resulted in better plan quality than using one. Additionally, two arcs were used based upon clinical experience with Esophageal cancer (EC) planning in King Abdullah Medical City (KAMC), where a single arc was found to be insufficient to achieve dose constraints. VMAT plan had a better homogeneity index (HI) and target coverage index (TCI) with the PTV and equivalent conformity index (see figures 4. A, 4. B and 4. C). Conformity index reports target dose coverage as a value between 0 and 1 . A value of 1 indicates an ideal plan with target coverage with no over/under-dosage of target sub-volumes, a CI value of 0 indicates the whole target volume is not covered by the therapeutic dose or the existence of a severe cold spot(s) in the target. Figure 5. A indicates that there were no significant differences in the conformity between the two modalities. The values of 3D-CRT were lower than VMAT, which is an indication of the improvement of the conformity in VMAT modality. This finding is consistent with pervious study that found VMAT capable of superior PTV conformity in Esophageal cancer (EC) treatment plans. An HI with a value close to 1 indicated better homogeneity. Figure 5. B illustrates the homogeneity index for both modalities, with VMAT plans showing significantly better PTV homogeneity. Additionally, TCI with a value close to 1 indicated relatively better target coverage. Figure 5. C shows the disparity in values between the two modalities, where the values of TCI were higher in some cases and lower in others. This is due to target coverage by prescribed dose. However, not all 
treatment plans were able to successfully meet each OAR dose constraint due to the close proximity of the PTV, while dose tolerance to critical structures was still maintained. VMAT achieved a better mean dose to central OARs: lungs, heart, liver, spinal canal, and kidneys.

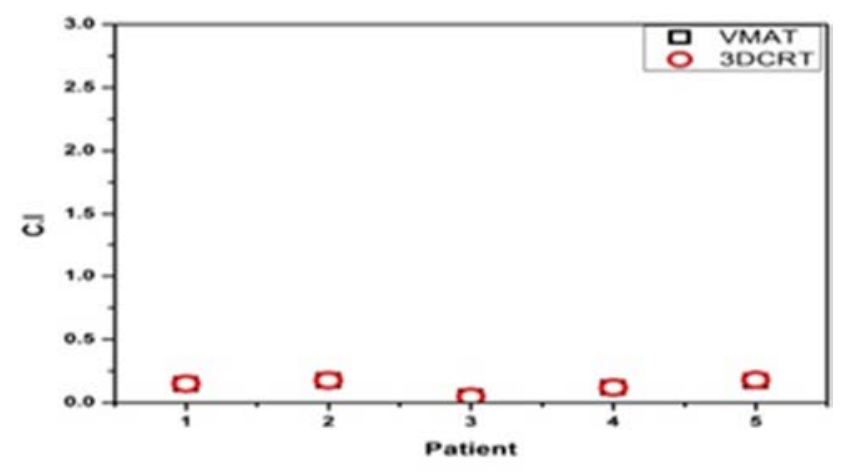

A

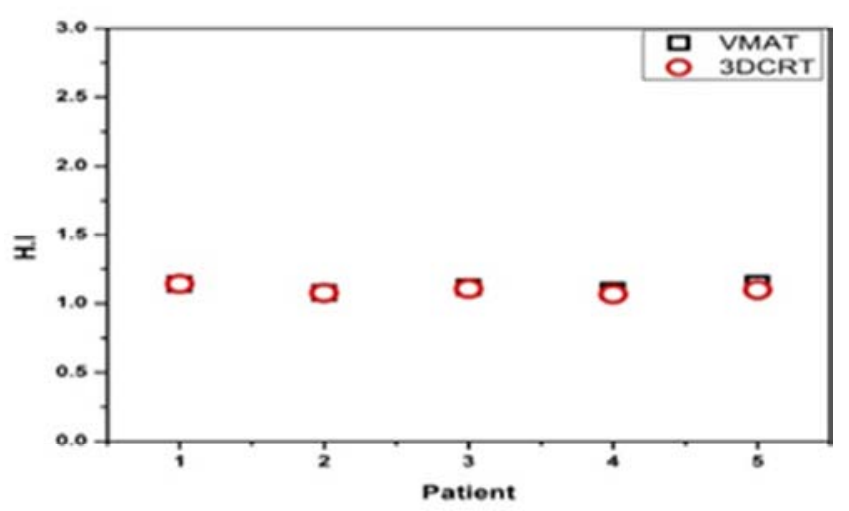

B

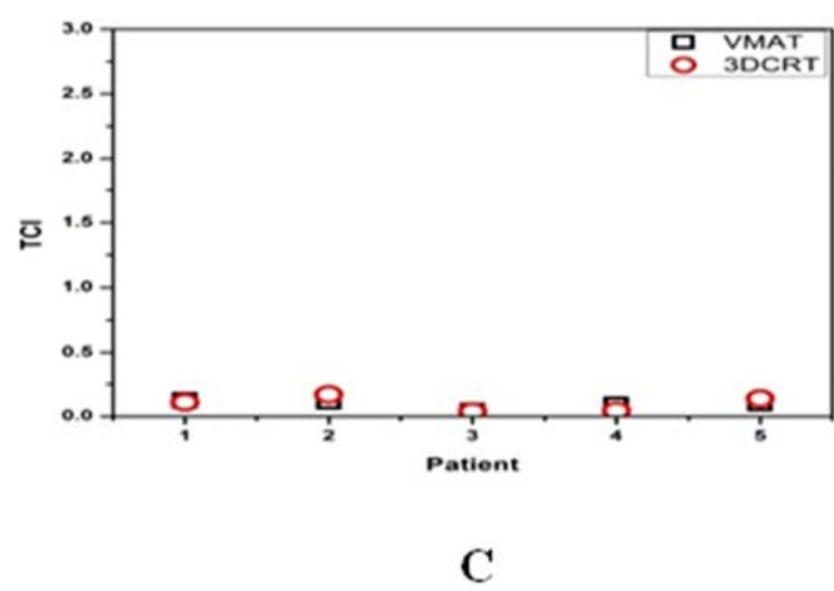

Figure 5. A histogram plot of $C$. $I(A), H I(B), T C I(C)$ for five esophagus cases.

\section{Conclusion}

Volumetric modulated arc therapy is a relatively new treatment technology that providing better conformity to the tumor, sparing healthy structures and better low-dose OAR sparing in the lungs and heart. This study showed that the VMAT is superior to 3D-CRT in term of PTV, conformity and homogeneity but not in term of doses to critical organs in some cases. Clinical preference for accepting the VMAT class solution over 3D-CRT treatment was preferred to be determined on a case by case basis. VMAT will be the treatment choice for tumor requiring PTV conformity and homogeneity that VMAT provides and suggests that VMAT class solution is the superior treatment option. VMAT was always superior to 3D-CRT in sparing the organs at risk or in PTV coverage during treatment of EC. However, VMAT offered an equivalent or better dose sparing of the lung and heart and a significant reduction in MUs per fraction. For upper EC patients, the PTV was T-shaped across the chest and neck; in these patients, VMAT provided a fairly uniform dose distribution. For patients with middle and lower EC in which the PTV involved more of the lung tissue, VMAT treatment had the potential to significantly increase the coverage of the lungs at low doses and the most uniform dose distribution. Compared with VMAT, 3D-CRT provided a lower mean dose and V5 of the lungs in patients with upper thoracic EC, but exhibited different advantages and disadvantages in patients with middle or lower thoracic EC. The major advantage of VMAT over 3D-CRT is the shorter treatment time. In conclusion, due to the ability of VMAT to generate highly conformal and efficient treatment plans that are clinically comparable to 3D-CRT, this study recommends VMAT be considered as a viable option for treatment different site of tumors.

\section{Acknowledgement}

We would like to thanks all staff members of the radiation oncology at KAMC (King Abdullah Medical City), especially Dr. Mohammad Dauod, Dr. Nasser Al-Dhaibani and Dr. Hussain Omar for their help and support in their areas of clinical expertise.

\section{References}

[1] Perez, Carlos A., and Luther W. Brady., "Radiation oncology: management decisions", Lippincott Williams \& Wilkins, 2011.

[2] Govindan, Ramaswamy, ed., "The Washington manual of oncology.", Lippincott Williams \& Wilkins, 2008.

[3] Parker, R. G., Janjan, N. A., and Selch, M. T., "Radiation oncology for cure and palliation." Springer Science \& Business Media, 2003.

[4] Purdy, James A., Carlos A. Perez, and Philip Poortmans., " Technical Basis of Radiation Therapy: Practical Clinical Applications.", Springer Berlin Heidelberg, 2012.

[5] International Commission On Radiation Units and Measurements. Prescribing, Recording and Reporting Photon Beam Therapy, ICRU Report50. Bethesda, MD: ICRU, 1993. 
[6] International Commission On Radiation Units and Measurements. Prescribing, Recording and Reporting Photon Beam Therapy, (Supplement to ICRU Report50). ICRU Report 62. Bethesda, MD: ICRU, 1999.

[7] Weiss, Elisabeth, and Clemens F. Hess., "The impact of gross tumor volume (GTV) and clinical target volume (CTV) definition on the total accuracy in radiotherapy", Strahlentherapie und Onkologie, 2003, 179. (1): 21-30.

[8] Vokes, Everett E., and Harvey M. Golomb, eds., "Oncologic therapies.", Springer Science \& Business Media, 2003.

[9] Perez, C. A., Purdy, J. A., Harms, W., Gerber, R., Graham, M. V., Matthews, J. W., and Simpson, J. R., "Three-dimensional treatment planning and conformal radiation therapy: preliminary evaluation.", Radiotherapy and Oncology, 1995, 36,(1): 32-43.

[10] Webb, S, "The Physics of three dimensional radiation Therapy: conformal therapy, radiosurgery and treatment planning.", Institute of Physics Publishing, Bristol, UK, 1993.

[11] Cheng, Chee-Wai, and Indra J. Das., "Treatment plan evaluation using dose-volume histogram (DVH) and spatial dose-volume histogram (zDVH)." International Journal of Radiation Oncology* Biology* Physics, 1999 43,(5): 11431150 .

[12] Mundt, Arno J., and John C. Roeske., " Intensity modulated radiation therapy: a clinical perspective.", PMPH-USA, 2005.

[13] Timmerman, Robert D., and Lei Xing., "Image-guided and adaptive radiation therapy.", Lippincott Williams \& Wilkins, 2012.

[14] Dybwad, Anniken. "Comparison of Dose Distributions resulting from IMRT and VMAT, and Assessment of MLC Leaf Positioning Errors.", 2013.

[15] Chao, KS Clifford, Smith Apisarnthanarax, and Gokhan Ozyigit, eds., "Practical essentials of intensity modulated radiation therapy.", Lippincott Williams \& Wilkins, 2005.

[16] Ishikura, Satoshi., "Quality assurance of radiotherapy in cancer treatment: toward improvement of patient safety and quality of care.," Japanese journal of clinical oncology, (2008), 38,(11): 723-729.

[17] Connell, Philip P., and Samuel Hellman., "Advances in radiotherapy and implications for the next century: a historical perspective." Cancer research, 2009, 69, (2): 383-392.

[18] $\mathrm{Wu}$, and Wing-cheung, "Inverse planning in three-dimensional conformal and intensity modulated radiotherapy.", Diss. The University of Hong Kong (Pokfulam, Hong Kong), 2004.

[19] Ezzell, G. A., Galvin, J. M., Low, D., Palta, J. R., Rosen, I., Sharpe, M. B., and Cedric, X. Y., "Guidance document on delivery, treatment planning, and clinical implementation of IMRT: report of the IMRT Subcommittee of the AAPM Radiation Therapy Committee.", (2003), Medical physics, 30.8: 2089-2115.

[20] Mancuso and Gordon Mark., "Evaluation of Volumetric Modulated Arc Therapy (VMAT) Patient Specific Quality Assurance.", Brigham Young University, 2011.

[21] Palma, D., Vollans, E., James, K., Nakano, S., Moiseenko, V., Shaffer, R., and Otto, K., "Volumetric modulated arc therapy for delivery of prostate radiotherapy: comparison with intensity-modulated radiotherapy and three-dimensional conformal radiotherapy." International Journal of Radiation Oncology Biology Physics, 2008, 72, (4): 996-1001.

[22] Salazar and Jessica Emily., "Optimization of RapidArc for Head-and-Neck Radiotherapy.", Diss. Duke University, 2011.

[23] Ong and Chin Loon., "Volumetric modulated arc therapy for stereotactic body radiotherapy: Planning considera-tions, delivery accuracy and efficiency.", Amsterdam: Vrije Universiteit, 2012.

[24] MacDonald, S. M., Ahmad, S., Kachris, S., Vogds, B. J., DeRouen, M., Gittleman, A. E., and Vlachaki, M. T., "Intensity modulated radiation therapy versus three-dimensional conformal radiation therapy for the treatment of high grade glioma: a dosimetric comparison." J Appl Clin Med Phys, 2007, 8, (2): 47-60.

[25] Cozzi, L., Dinshaw, K. A., Shrivastava, S. K., Mahant-shetty, U., Engineer, R., Deshpande, D. D., and Fogliata, A., "A treatment planning study comparing volumetric arc modulation with RapidArc and fixed field IMRT for cervix uteri radiotherapy.", Radiotherapy and Oncology, 2008, 89, (2): 180-191.

[26] Verbakel, W. F., Cuijpers, J. P., Hoffmans, D., Bieker, M., Slotman, B. J., and Senan, S., "Volumetric intensi-tymodulated arc therapy vs. conventional IMRT in head-andneck cancer: a comparative planning and dosi-metric study.", International Journal of Radiation Oncolo-gy* Biology* Physics, 2009, 74, (1): 252-259.

[27] Zheng, B. M., Dong, X. X., Wu, H., Duan, Y. J., Han, S. K., and Sun, Y., "Dosimetry comparison between volumetric modulated arc therapy with RapidArc and fixed field dynamic IMRT for local-regionally advanced nasopharynge-al carcinoma.", Chinese Journal of Cancer Research, 2011, 2, (4): $259-264$.

[28] Peszynska-Piorun, Magdalena, Julian Malicki, and Wojciech Golusinski., "Doses in organs at risk during head and neck radiotherapy using IMRT and 3D-CRT.", Radi-ology and oncology, 2012, 4, (4): 328-336.

[29] Holt, A., Van Gestel, D., Arends, M. P., Korevaar, E. W., Schuring, D., Kunze-Busch, M. C., and van VlietVroegindeweij, C., "Multi-institutional comparison of volumetric modulated arc therapy vs. intensity-modulated radiation therapy for head-and-neck cancer: a planning study.", Radiation Oncology, 2013, 8,(1): 26.

[30] Nguyen, K., Cummings, D., Lanza, V. C., Morris, K., Wang, C., Sutton, J., and Garcia, J., "A dosimetric comparative study: Volumetric modulated arc therapy vs intensi-tymodulated radiation therapy in the treatment of nasal cavity carcinomas.", Medical Dosimetry, 2013, 38,(3): 225-232.

[31] Nabavizadeh, N., Simeonova, A. O., Waller, J. G., Romer, J. L., Monaco, D. L., Elliott, D. A., and Holland, J. M., "Volumetric-modulated arc radiotherapy for pancreatic malignancies: Dosimetric comparison with sliding-window intensity-modulated radiotherapy and 3-dimensional conformal radiotherapy.", Medical Dosimetry, 2014, 39, (3): 256-260.

[32] Zhang, Q., Li Yu, X., Hu, W. G., Chen, J. Y., Wang, J. Z., Ye, J. S., and Guo, X. M., "Dosimetric comparison for volumet-ric modulated arc therapy and intensity modulated radio-therapy on the left-sided chest wall and internal mammary nodes irradiation in treating post-mastectomy breast can-cer.", Radiology and Oncology, 2012. 
[33] Patil, S. S., May, K. S., Hackett, R. A., Hales, L. D., Van Benthuysen, L. T., Malik, N. K., and Warren, G. W., "A Comparison of VMAT, IMRT, and 3DCRT in the Treatment Planning of Patients with Distal Esophageal Cancer.", International Journal of Radiation Oncology* Biology* Physics, 81.2 (2011): S324-S325.

[34] Planning Your Treatment: [Cited May, 2013] Available at: http://www.macmillan.org.uk/Cancerinformation/Cancertreat ment/Treatmenttypes/Radiotherapy/Beforetreatment/Radiother apymasks.aspx

[35] Aird, E. G. A., and J. Conway., "CT simulation for radiotherapy treatment planning." The British journal of radiol-ogy, 2002, 75,(900): 937-949.

[36] Alvarez-Moret, J., Pohl, F., Koelbl, O., and Dobler, B., "Evaluation of volumetric modulated arc therapy (VMAT) with Oncentra Master Plan for the treatment of head and neck cancer.", Radiation Oncology, 2010, 5, (110): 110.

[37] Kuo, Y. C., Chiu, Y. M., Shih, W. P., Yu, H. W., Chen, C. W., Wong, P. F., and Hwang, J. J., "Volumetric intensi-tymodulated Arc (RapidArc) therapy for primary hepato-cellular carcinoma: comparison with intensity-modulated radiotherapy and 3-D conformal radiotherapy.", Radiation Oncology, 2011, 6: 76 .

[38] Yi, B. Y., Ahn, S. D., Kim, J. H., Lee, S. W., and Choi, E. K., "IMRT optimization with pseudo-biologic objective function.", 2002.

[39] Jemal A, Siegel R, Ward E, et al. Cancer statistics, 2008. CA Cancer J Clin 2008; 58: 71-96.

[40] Kaifi JT, Gusani NJ, Jiang Y, et al. Multidisciplinary management of early and locally advanced esophageal cancer. J Clin Gastroenterol 2011; 45: 391-399.

[41] Lin CY, Huang WY, Jen YM, et al. Dosimetric and efficiency comparison of high-dose radiotherapy for esophageal cancer: volumetric modulated arc therapy versus fixed-field intensitymodulated radiotherapy. Dis Esophagus 2014; 27: 585-590.
[42] Cooper JS, Guo MD, Herskovic A, et al. Chemoradiotherapy of locally advanced esophageal cancer: long-term follow-up of a prospective randomized trial (RTOG 85-01). Radiation Therapy Oncology Group. JAMA 1999; 281: 1623-1627.

[43] Minsky BD, Pajak TF, Ginsberg RJ, et al. INT 0123 (Radiation Therapy Oncology Grou (94-95) phase III trial of combined-modality therapy for esophageal cancer: high-dose versus standard-dose radiation therapy. J Clin Oncol 2002; 20; $1167-1174$

[44] Chandra A, Guerrero TM, Liu HH, et al. Feasibility of using intensity-modulated radiotherapy to improve lung sparing in treatment planning for distal esophageal cancer. Radiother Oncol 2005; 77: 247-253.

[45] Welsh J, Palmer MB, Ajani JA, et al. Esophageal cancer dose escalation using a simultaneous integrated boost technique. Int J Radiat Oncol Biol Phys 2012; 82: 468-474.

[46] Yu CX. Intensity-modulated arc therapy with dynamic multileaf collimation: an alternative to tomotherapy. Phys Med Biol 1995; 40: 1435-1449.

[47] Bertelsen A, Hansen CR, Johansen J, et al. Single Arc Volumetric Modulated Arc Therapy of head and neck cancer. Radiother Oncol 2010; 95: 142-148.

[48] Verbakel WF, Cuijpers JP, Hoffmans D, et al. Volumetric intensity-modulated arc therapy vs. conventional IMRT in head-and-neck cancer: a comparative planning and dosimetric study. Int J Radiat Oncol Biol Phys 2009; 74: 252-259.

[49] Shaffer R, Morris WJ, Moiseenko V, et al. Volumetric modulated Arc therapy and conventional intensity-modulated radiotherapy for simultaneous maximal intraprostatic boost: a planning comparison study. Clin Oncol 2009; 21: 401-407.

[50] Hawkins MA, Bedford JL, Warrington AP, et al. Volumetric modulated arc therapy planning for distal oesophageal malignancies. Br J Radiol 2012; 85: 44-52. 